\title{
Effect of the endothelial nitric oxide synthase Glu298Asp polymorphism on endothelial function and lipid profile in healthy subjects
}

\author{
A. K. Thompson ${ }^{1}$, K. J. Newens ${ }^{1}$, K. G. Jackson ${ }^{1}$, S. Todd ${ }^{2}$ and C. M. Williams ${ }^{1}$ \\ ${ }^{1}$ Hugh Sinclair Unit of Human Nutrition, Department of Food and Nutritional Sciences, University of Reading, Reading \\ RG6 6AP, UK and ${ }^{2}$ Department of Mathematics and Statistics, University of Reading, Reading RG6 6AX, UK
}

The Glu298Asp endothelial nitric oxide synthase (eNOS) polymorphism has been associated with an increased risk of CHD ${ }^{(1)}$, and there is some evidence of a less favourable lipid profile ${ }^{(2,3)}$ and reduced endothelial function in Asp298 carriers ${ }^{(3,4)}$. However, many studies have genotyped retrospectively, resulting in low numbers of homozygous Asp298 subjects. This study investigated potential effects of the Glu298Asp polymorphism on endothelial function and lipid profile in prospectively genotyped subjects.

Two groups of healthy individuals homozygous for Asp298 ( $n$ 30) and Glu298 ( $n$ 30) were balanced for gender, age (mean 27.9 years, range $18-65$ years) and BMI (mean $23.2 \mathrm{~kg} / \mathrm{m}^{2}$, range $18-32 \mathrm{~kg} / \mathrm{m}^{2}$ ). Subjects had their endothelial function measured using flowmediated dilatation (FMD) and laser Doppler imaging with iontophoresis. A fasting blood sample was taken for the analysis of TAG, total- and HDL-cholesterol (HDL-C) and NEFA levels. LDL cholesterol (LDL-C) was calculated using the Friedewald formula. The fatty acid composition of NEFA and phosphatidylcholine (PC) were used as biomarkers of dietary fat intake.

There were no differences between genotypes for TAG or total cholesterol, but Asp298 subjects had lower HDL-C levels and higher TC:HDL-C and LDL-C:HDL-C ratios (all $P \leq 0.022$, Table 1). Female Asp298 also had higher NEFA $(P=0.048)$ and LDL-C $(P=0.052)$ levels than their Glu298 counterparts. There were no differences between genotypes for any measure of endothelial function or in the percentage weight of SFA or long-chain $n-3$ PUFA in either NEFA or PC fractions. However, there was a strong correlation between NEFA long-chain $n-3$ PUFA levels and FMD response $(r=0.905, P<0.001)$ in Asp298 females.

These findings provide additional evidence for the mechanism(s) underlying the increased CHD risk in Asp298 individuals. They support previously reported associations between long-chain $n$-3 PUFA intake and FMD in Asp298 subjects ${ }^{(5)}$, which raises the possibility that dietary interventions such as increasing LC $n-3$ PUFA intake may improve endothelial function and thus reduce the increased CHD risk, particularly in Asp298 females.

Table 1. Lipid profile of groups based on Glu298Asp polymorphism

\begin{tabular}{|c|c|c|c|c|c|c|c|c|c|}
\hline & \multicolumn{4}{|c|}{ Females } & \multicolumn{4}{|c|}{ Males } & \multirow{3}{*}{$\begin{array}{c}P \text {-value (between } \\
\text { genotypes) }\end{array}$} \\
\hline & \multicolumn{2}{|c|}{ Asp289 } & \multicolumn{2}{|c|}{ Glu298 } & \multicolumn{2}{|c|}{ Asp289 } & \multicolumn{2}{|c|}{ Glu298 } & \\
\hline & mean & SEM & mean & SEM & mean & SEM & mean & SEM & \\
\hline NEFA $(\mu \mathrm{mol} / \mathrm{l})$ & $640 *$ & 31 & $550^{*}$ & 29 & 479 & 34 & 527 & 37 & 0.554 \\
\hline LDL-C (mmol/l) & $2.92 *$ & 0.66 & $2.44 *$ & 0.46 & 2.77 & 0.69 & 2.70 & 0.74 & 0.140 \\
\hline HDL-C (mmol/l $)$ & $1.52 *$ & 0.10 & $1.84 *$ & 0.10 & $1.15^{*}$ & 0.15 & $1.36^{*}$ & 0.08 & 0.011 \\
\hline TC:HDL-C ratio & $3.3^{*}$ & 0.2 & $2.6^{*}$ & 0.1 & 3.8 & 0.2 & 3.5 & 0.2 & 0.022 \\
\hline LDL-C:HDL-C ratio & $2.0^{* *}$ & 0.2 & $1.4 * *$ & 0.1 & 2.4 & 0.2 & 2.1 & 0.2 & 0.010 \\
\hline
\end{tabular}

$N \geq 13$ for all subgroups. Difference between genotypes within a gender: $* P \leq 0.05 ; * * P \leq 0.01$.

The authors gratefully acknowledge funding from BBSRC, Unilever and FRST (New Zealand) and assistance from Agnieszka Przemska, Dafni Vasilopoulou and Alice Turner.

1. Casas JP et al. (2006) Am J Epidemiol 164(10), 921-935.

2. Ferguson JF et al. (2010) Atherosclerosis 211(2), 539-544.

3. Imamura A et al. (2008) Eur J Endocrinol 158(2), 189-195.

4. Paradossi U et al. (2004) Stroke 35(6), 1305-1309.

5. Leeson CPM et al. (2002) Circ Res 90(11), 1153-1158. 